\title{
Working together: Paramedic students' and student midwives' experiences of interprofessional learning
}

Feltham, C., Foster, J., Davidson, T., \& Ralph, S. (2016). Student midwives and paramedic students' experiences of shared learning in pre-hospital childbirth. Nurse Education Today, 41(1), 73-78.

The World Health Organisation (WHO, 2010) recognises that interprofessional learning (IPL) in education institutes is an innovative strategy in mitigating the health workforce crisis and improving patient outcomes. Almost 50 years of evidence has enabled the WHO and its partners to conclude that IPL is a part of education that will shape an effective and collaborative workforce, which will ultimately support local health needs. Furthermore Barr, Helme and D'Avray (2014) highlighted that IPL should be embedded across university programmes, in order to maximise the learning experiences of healthcare students.

The authors identified a lack of research into IPL between student midwives and paramedic students. The two professions work closely together in the management of pre-hospital obstetric births and emergencies, and the authors believe a greater understanding of each other's roles is paramount to enable optimal patient care.

Twenty-five student midwives and 31 paramedic students participated in an IPL workshop lasting 6 hours. The same workshop was conducted on two separate days to accommodate effective group sizes. The programme incorporated a brief introduction to IPL and communication; opportunities to discuss in small groups their understanding of each other's role and scope of practice; equipment familiarisation sessions with participants taking it in turns to demonstrate their profession specific equipment; and participation in high fidelity pre-hospital obstetric scenarios, enabling students from both professions to work together.

Following the workshop, five videoed focus groups were carried out; two with paramedic students and three with student midwives. Qualitative data analysis software was used to collate the transcripts from the focus groups. Data were analysed using a process of thematic coding and categorisation enabling the emergence and identification of four key themes.

Theme 1 - 'Understanding of each other's roles and responsibilities'. The students felt after the IPL workshop they had a better understanding of each other's roles and responsibilities; improved role identification in scenarios; a better recognition of who was the clinical lead; and insight into limitations within each profession.

Theme 2 - 'Value of interprofessional learning'. This included recognition of the value of simulation and peer assisted learning; improved awareness of medical abbreviations commonly used by each profession; an appreciation of the importance of record keeping; improved understanding of the different equipment.

Theme 3 - 'Organisation of the day'. Participants liked the opportunity to work in small groups; the structure and timing of the day and individual activities were seen as appropriate; simulation based learning was viewed positively; but participants would have appreciated a stronger conclusion/summary to the day. 
Theme 4 - 'Further/future learning'. The students recognised that they would benefit from further IPL with mixed groups of health and social care professionals. The student midwives wanted more collaborative learning with student social workers and student physiotherapists; and the paramedic students wanted to work with other emergency services as well as social workers, end of life care teams, and mental health clinicians.

The authors recognised that students may have limited knowledge of their own profession's role and scope of practice and that this could be a limitation. Furthermore due to the sample size, the findings are not representative of all student midwives and paramedic students.

To conclude, the students enjoyed interacting with each other and learning about the differences and similarities between their professions. The workshops fostered a positive relationship between participants which, in turn, could influence the interaction of these two professions in the healthcare setting to enhance patient care. The importance of IPL being embedded within higher education for paramedic students is essential and the findings of this study add strength to this argument.

\section{$\underline{\text { References }}$}

Barr, H., Helme, M., \& D'Avray, L. (2014). Review of Interprofessional Education in the United Kingdom. Fareham: CAIPE.

World Health Organisation (2010). Framework for Action on Interprofessional Education and Collaborative Practice. Geneva: World Health Organisation.

Richard Ward, Clinical Education facilitator, University of Greenwich 